\title{
Theoretical and experimental study of ScAlN/Sapphire structure based SAW sensor
}

\author{
F. Bartoli, M. Moutaouekkil, J. Streque, P. Pigeat, \\ S. Hage-Ali, P. Boulet, H. M'Jahed and O. Elmazria, \\ Institut Jean Lamour UMR 7198 \\ Université de Lorraine - CNRS \\ 54506 Vandœuvre-lès-Nancy, France \\ F. Bartoli, T. Aubert \\ LMOPS EA 4423 \\ CentraleSupélec - Université de Lorraine \\ 57070 Metz, France
}

\author{
Sergei Zhgoon \\ National Research University "MPEI" \\ 14, Krasnokazarmennaya \\ 121351, Moscow Russia \\ O. Bou Matar, A. Talbi \\ Joint International Laboratory LIA LICS \\ Univ. Lille, CNRS, Centrale Lille, ISEN, Univ. \\ Valenciennes, UMR 8520 - IEMN \\ 59651 Villeneuve d'Ascq, France
}

\begin{abstract}
Several SAW devices based on $\mathrm{Sc}_{0.1} \mathbf{A l}_{0.9} \mathrm{~N} / \mathrm{Sapphire}$ bilayer structures were fabricated using various wavelengths and film thicknesses. The acoustic velocity, electromechanical coupling coefficient and temperature coefficient of frequency (TCF) of each device was then measured and the results were compared with calculations using several sets of elastic, piezoelectric and dielectric constants available in the literature. We have shown that the accuracy of available constants is not enough to permit a reliable optimization and design of SAW devices for signal processing and sensors applications.
\end{abstract}

\section{Keywords-Temperature sensor, SAW, ScAlN}

\section{INTRODUCTION}

Thanks to its high acoustic phase velocity, low motional resistance, high thermal conductivity, and CMOS-compatibility $[1,2]$, AlN thin films have attracted attention as piezoelectric material for RF-MEMS including bulk and surface acoustic wave devices. Moreover, AIN is chemically inert and shows a high temperature stability. Indeed it is an intrinsically-poled, nonferroelectric material (no Curie point) which has been reported to retain its piezoelectric properties at temperatures above $1000^{\circ} \mathrm{C}$ [3]. However, the low electromechanical coupling coefficient $\left(\mathrm{K}^{2}\right)$ and piezoelectric constant $\left(\mathrm{d}_{33}\right)$ of $\mathrm{AlN}$ thin compared to PZT and $\mathrm{ZnO}$ thin films, limits its wide applications in sensors, wide band filters, and MEMS including for energy harvesting.

Recently, several studies have shown that the addition of $\mathrm{Sc}$ to form $\mathrm{Sc}_{\mathrm{x}} \mathrm{Al}_{1-\mathrm{x}} \mathrm{N}$ strongly increases the measured piezoelectric coefficients of ScAlN alloys, until reaching a phase transition to the non-polar rocksalt-type structure $[1,2]$ $(x=0.43)$. It was reported that an enhancement of $400 \%$ of $\mathrm{d}_{33}$ is obtained for $\mathrm{Sc}_{0.43} \mathrm{Al}_{0.57} \mathrm{~N}$ alloys compared to pure AlN [4]. Moreover, the ScAlN has all the advantages of the AlN mentioned below. Because of its strong electromechanical coupling and improved piezoelectricity, ScAlN allows to widen the field of applications and especially that of sensors. Note also that the expected energy harvesting factor of merit $\left(F O M=\frac{e_{31}^{2}}{\varepsilon 0 \varepsilon \mathrm{r}}\right)$ for $\mathrm{Sc}_{0.43} \mathrm{Al}_{0.57} \mathrm{~N}(60 \mathrm{GPa})$ is larger than those of PZT (24GPa) or $\mathrm{ZnO}$ (10.8GPa) [5].
The aim of this work is to develop a wireless, batteryless and packageless acoustic wave sensor based on the three layers structure AlN/ScAlN/Sapphire. In order to develop fully operational sensors and optimize their design according to the targeted performance, the full and accurate physical constants of the materials considered are a prerequisite. If theses constants are now well established for Sapphire and AlN, it is not the case for ScAlN. Several sets of constants could be found in literature [7-11] and these constants are of course depending on the concentration of scandium in the film. These constants, determined by different experimental methods or by calculation, show a strong scatter, making the choice of the accurate set difficult. Consequently, and in order to determine the most suitable set of constants, we first studied the bilayer structure ScAlN/Sapphire and compared experimental results with calculated ones. The selected set is then used to optimize the whole set constants AIN/ScAIN/Sapphire structure.

\section{MODELING DETAILS AND METHOD}

Phase velocity of ScAlN/Sapphire structures have been determined using the recently reported Legendre and Laguerre polynomial approach of wave propagation in layered magnetoelectro-elastic structures by which is a flexible and computationally efficient alternative to the classical solution of transcendental equations [12]. For $\mathrm{S}_{11}$ coefficient calculation, a numerical model in Comsol Multiphysics using general partial derivative equations interface. A half period of an infinite IDT with periodic boundary conditions in the propagation direction is used. Dispersion curves of acoustic velocity were calculated versus ScAlN film thickness, and for various Sc concentration in the film.

Like AlN, ScAlN is part of the family of hexagonal piezoelectric crystals (class $6 \mathrm{~mm}$ ) and can be defined by 10 independent material constants as follows:

Elastic stiffness: $\mathbf{C}_{\mathbf{1 1}}, \mathbf{C}_{\mathbf{1 2}}, \mathbf{C}_{\mathbf{1 3}}, \mathbf{C}_{\mathbf{3 3}}, \mathbf{C}_{\mathbf{4 4}}$, with $\mathbf{C}_{\mathbf{6 6}}=\frac{C_{11}-C_{12}}{2}$

Piezoelectric constants: $\mathbf{e}_{\mathbf{1 5}}, \mathbf{e}_{\mathbf{3 1}}, \mathbf{e}_{\mathbf{3 3}}$

Dielectric constants: $\varepsilon_{11}, \varepsilon_{33}$,

To optimize the considered structure and thus the design of acoustic wave devices, the full and accurate material constant

This work has been supported by the grant ANR-15-CE08-0015 SALSA, by the Region Grand-Est, by the French PIA project "Lorraine Université d'Excellence", ref. ANR-15-IDEX-04-LUE; and by the Ministry of Science and Education of Russian Federation 8.6108.2017/6.7. 
sets are required. Table 1 summarizes the main data collected in published works concerning the ScAlN. It has been demonstrated by calculation [10] and experimentally [9] that the elastic constants $C_{i j}$ vary linearly with the concentration of
Scandium. In order to obtain a complete set of constants for lower concentrations, we have determined these constants by extrapolation considering those of the pure $\mathrm{AlN}(\mathrm{x}=0 \%)$ and the available ones (generally $\mathrm{x}=43 \%$ ).

TABLE I. PHYSICAL CONSTANTS OF CONSIDERED MATERIAL

\begin{tabular}{|c|c|c|c|c|c|}
\hline Material & Elastic stiffness (GPa) & Piezoelectric constants $\left(\mathrm{C} / \mathrm{m}^{2}\right)$ & Dielectric constants & Density $\left(\mathrm{kgm}^{-3}\right)$ & Ref \\
\hline & $\mathrm{C}_{11}, \quad \mathrm{C}_{12}, \quad \mathrm{C}_{13}, \quad \mathrm{C}_{33}, \quad \mathrm{C}_{44}, \mathrm{C}_{66}$ & $\mathbf{e}_{15}, \mathbf{e}_{31}, \mathbf{e}_{33}$ & $\varepsilon_{11}, \varepsilon_{33}$ & $\rho$ & \\
\hline AlN & $345,125,120395,118,110$ & $-0.48,-0.58,1.55$ & $8,9.5$ & 3260 & [6] \\
\hline \multirow{5}{*}{$\mathrm{Sc}_{43 \%} \mathrm{AlN}_{57 \%}$} & $234.5,101.4,128.4,267.4,39.6,66.6$ & $1.03,-0.886,0.863$ & $24.1,12.3$ & 3601 & [7] \\
\hline & $169,61.2,58.8,211,51.5,53.9$ & $-1.31,-1.58,4.42$ & $30.5,30.28$ & 3760 & [8] \\
\hline & $282.4,136.5,125.2,169.6,98.1,98.1$ & $-0.294,-0.516,2.72$ & $30.5,30.28$ & 3760 & [9] \\
\hline & $313.7,150,139.2,197,108.6,108.6$ & $-0,317,-0,722,2,73$ & $30.5,30.28$ & 3760 & [10] \\
\hline & $293,146,130,184,100,74.7$ & $-1.31,-1.58,4.42$ & $30.5,30.28$ & 3760 & [11] \\
\hline
\end{tabular}

\section{EXPERIMENTAL}

\section{A. ScAlN deposition}

Scandium Aluminum Nitride thin films were deposited by reactive magnetron sputtering. $10 \%$ scandium and $18 \%$ scandium thin films have been made with single composite $\mathrm{Sc} / \mathrm{Al}$ targets, respectively composed of $12.5 \%$ and $25 \%$ of Scandium. The films compositions have been measured by Energy Dispersive X-Ray Spectroscopy (EDXS). Structural properties such as microstructure and nanostructure have been determined by $\theta-2 \theta$, rocking-curve and pole figures X-Rays Diffraction (XRD) measurements and by Transmission Electron Microscopy (TEM) measurements. The $\mathrm{Sc}_{0.1} \mathrm{Al}_{0.9} \mathrm{~N}$ and $\mathrm{Sc}_{0.18} \mathrm{Al}_{0.82} \mathrm{~N}$ thin films shows strong (002) orientation, with high texturing which guarantees good piezoelectric properties which in principle enables to obtain good piezoelectric properties.

\section{B. SAW devices fabrication}

Several SAW devices based on ScAlN/Sapphire bilayer structures were fabricated using photolithography and chemical etching process. SAW devices consist in asynchronous resonator. Aluminum Interdigital transducer (IDT) and reflectors were fabricated by conventional contact ultraviolet (UV) photolithography. Resonators consist in 100 IDT pairs and 200 reflectors on each side. Two spatial periods $(\lambda=6.5$ and $13 \mu \mathrm{m})$ and three ScAlN thicknesses (1.6, 2.23 and $2.76 \mu \mathrm{m})$ were considered. Thus, six values of relative thickness $\left(h_{\text {ScAIN }}=h / \lambda\right)$ are obtained, leading to plot dispersion curves of velocity, $\mathrm{K}^{2}$ and temperature coefficient of frequency (TCF).

\section{RESULTS AND DISCUSION}

As an example, figure 1 shows the wide range frequency response of a SAW resonator fabricated with $(\lambda=6.5 \mu \mathrm{m}$ and $\left.\mathrm{h}_{\text {ScAIN }}=2.76 \mu \mathrm{m}\right)$. We can observe the Rayleigh wave at 729 $\mathrm{MHz}$ and its $2^{\text {nd }}$ and $3^{\text {rd }}$ mode at $1025 \mathrm{MHz}$ and $1566 \mathrm{MHz}$. The larger coupling is obtained for the $3^{\text {rd }}$ mode $\left(\mathrm{K}^{2}=1.7 \%\right)$ while $0.77 \%$ and $0.49 \%$ were obtained respectively for modes 1 and 2 . We note that for lower relative thicknesses of ScAlN, only the mode 1 of Rayleigh wave is generated. In the following part our study will be focused only on this mode. The phase velocities (v) were determined from the resonance frequency fr $(v=\lambda . f r)$. The experimental values of $\mathrm{K}^{2}$ were determined using resonance (fr) and antiresonance (fa) frequencies determined from admittance $Y$ plot and using formula $K^{2}=1-\left(\mathrm{fr} / \mathrm{fa}^{2}\right.$. All devices were characterized versus temperature leading to determination of TCF values.

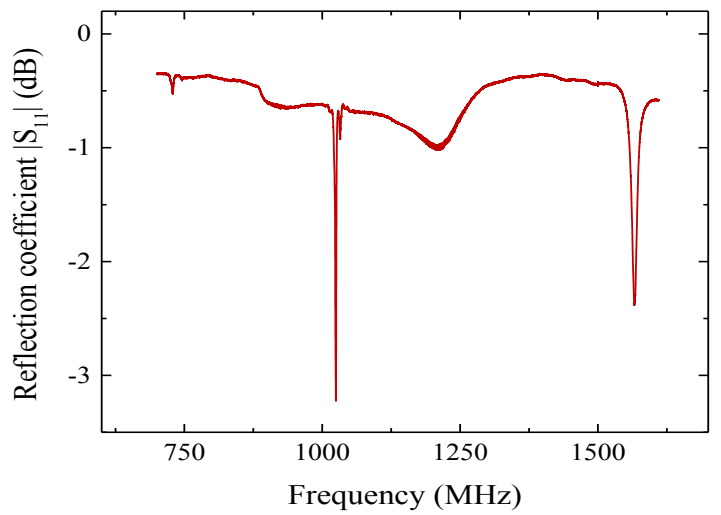

Fig. 1. Frequency response of a AlN/Sapphire SAW resonator fabricated with $\mathrm{hScAlN}=2.76 \mu \mathrm{m}$ and $\lambda=6.5 \mu \mathrm{m}$.

Figure 2 shows the dispersion curves of the phase velocity for a scandium concentration of $43 \%$. As reference curve, dispersion curves of pure AlN were also plotted in the same graphs. As expected, the calculated values are strongly dependent on the considered set of constants. To determine the most accurate set, we plot in the same graph the experimental value of velocity measured for samples with scandium concentration of $10 \%$ and the calculated velocity values (Fig. 3). On can observe that the measured velocities are lower than the calculated ones regardless of the constant set used. The experimental data are closer to those calculated for $43 \%$ of scandium with Zhang set [10]. These differences could be explained by cracks at the surface due to the high temperature deposition of the ScAIN thin film, which could decrease the SAW velocity. Further investigations are made for more informations. If the data published by Konno remains the closest to the experimental ones, they are nevertheless not suitable for making a reliable optimization. 


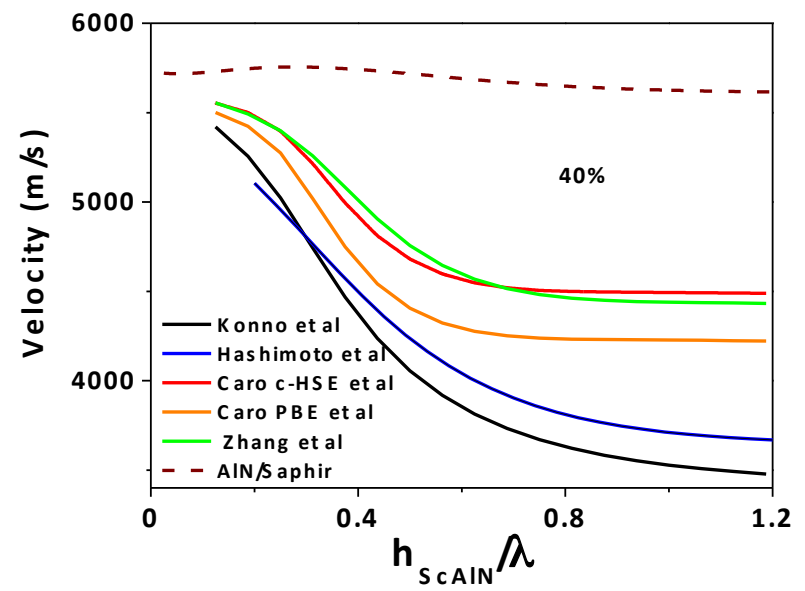

Fig. 2. Dispersion curve of velocity calculated for $\mathrm{Sc}_{0.43} \mathrm{Al}_{0.57} \mathrm{~N} /$ Sapphire structure using five different sets of physical constants.

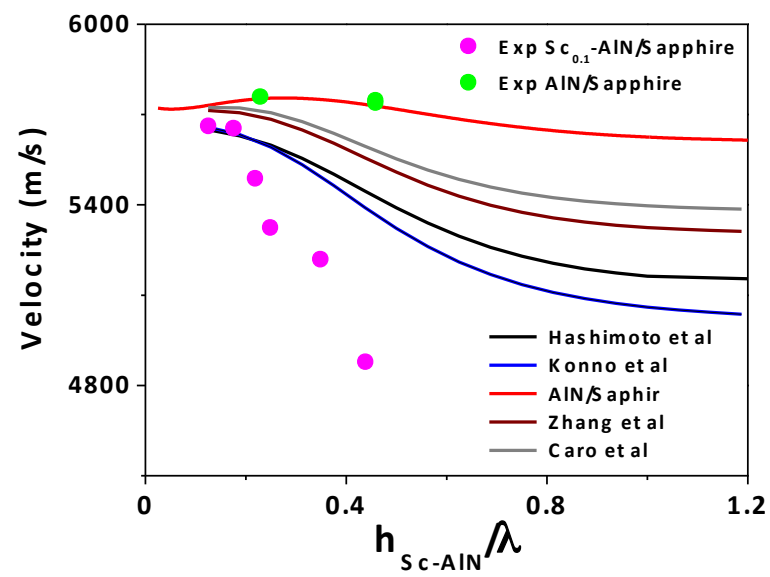

Fig. 3. Dispersion curve of velocity measured and calculated for $\mathrm{Sc}_{0.1} \mathrm{Al}_{0.9} \mathrm{~N} /$ Sapphire structure.

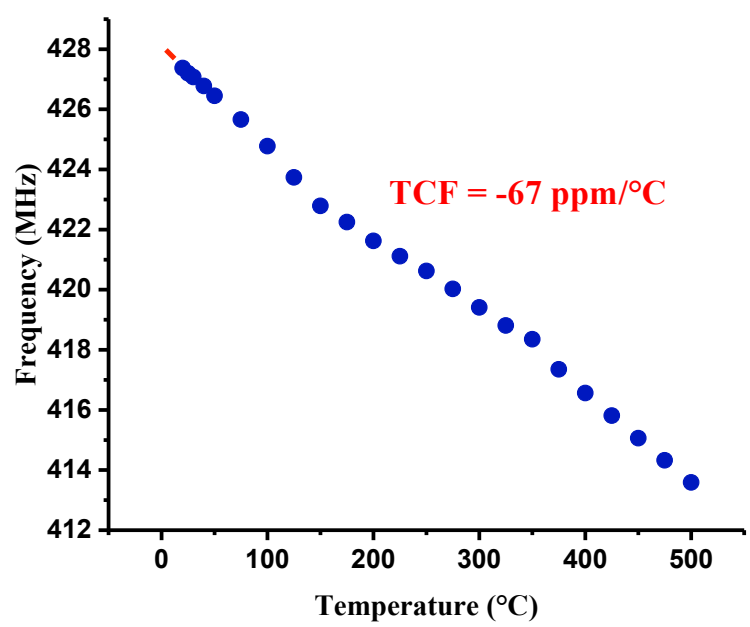

Fig. 4. Measured TCF for a $2.76 \mu \mathrm{m}$ ScAlN thin film on Sapphire substrate, with $\lambda=13 \mu \mathrm{m}$.
TCF values were also determined for various devices made with $\mathrm{Sc}_{0.1} \mathrm{Al}_{0.9} \mathrm{~N}$. These values are depending on both relative film thickness and scandium concentration. For characterized devices the TCF is ranging from $-110 \mathrm{ppm} /{ }^{\circ} \mathrm{C}$ to $-40 \mathrm{ppm} /{ }^{\circ} \mathrm{C}$. For instance, Figure 4 shows a TCF of $-67 \mathrm{ppm} /{ }^{\circ} \mathrm{C}$, for temperatures between $20^{\circ} \mathrm{C}$ and $500^{\circ} \mathrm{C}$. Therefore, the $\mathrm{Sc}_{0.1} \mathrm{Al}_{0.9} \mathrm{~N}$ shows a good sensitivity of the temperature, and it is able to operate up to $500^{\circ} \mathrm{C}$. Since the IDT were made with aluminum, the robustness of the film to higher temperatures has not been tested yet. Moreover, the advantage of such structure is that the TCF and thus the sensitivity of the temperature sensor, could be adjusted to match application requirements by controlling the film thickness and/or the Sc to Al ratio.

\section{CONCLUSION}

Highly textured Scandium Aluminum Nitride thin films were deposited by reactive magnetron sputtering on sapphire substrates. The film quality was demonstrated by XRD and TEM measurements and by the performances of the fabricated SAW resonators. The comparison of the dispersion curves of velocity calculated and determined experimentally, shows a strong divergence thus suggesting the inaccuracy of the physical constants available in the literature for the ScAlN. The unavailability of elastic constants thermal properties does not make it possible to determine the TCF of the ScAlN/Sapphire structure by calculation.

\section{REFERENCES}

[1] M. Akiyama, T. Kamohara, K. Kano, A. Teshigahara,Y. Takeuchi, and N. Kawahara, Advanced Materials 21, 593(2009).

[2] S. Zhang, D. Holec, W. Y. Fu, C. J. Humphreys, and M. A. Moram, Journal of Applied Physics 114, 133510 (2013).

[3] T. Aubert, J. Bardong, O. Legrani, O. Elmazria, M. B. Assouar, G. Bruckner ; J. of Applied Physics, Vol. 114, art. Nu 014505; (2013)

[4] M. Akiyama, K. Umeda, A. Honda, and T. Nagase, Applied Physics Letters 102, 021915 (2013).

[5] P. M. Mayrhofer, C. Rehlendt, M. Fischeneder, M. Kucera, E. Wistrela, A. Bittner, and U. Schmid; JOURNAL OF Microelectromechanical Systems, Vol. 26, pp 102-112, (2017)

[6] K. Tsubouchi, K. Sugai and N. Mikoshiba, Proc. IEEE Ultrasonics Symp., pp. 375-380, 1981.

[7] A. Konno, M. Kadota, J. Kushibiki, Y. Ohashi, M. Esashi, Y. Yamamoto, S. Tanaka; 2014 IEEE International Ultrasonics Symposium Proceedings ; pp 273-276 (2014).

[8] Ken-ya Hashimoto, S. Sato, A. Teshigahara, T. Nakamura and K. Kano; IEEE Transactions on Ultrasonics, Ferroelectrics, and Frequency Control , vol. 60, no. 3, pp 637 - 642, March (2013).

[9] M.A. Caro, S. Zhang, M. Ylilammi, T. Riekkinen, M. A Moram, O. Lopez-Acevedo, J. Molarius and T. Laurila; J. Phys. Condens. Matter 27 245901);

[10] S. Zhang, W. Y. Fu1, D. Holec, C. J. Humphreys and M. A. Moram; Journal of Applied Physics 114, 243516, pp 1-6 (2013).

[11] G. Carlotti, J. Sadhu , F. Dumont; IEEE International Ultrasonics Symposium (IUS), 1037, pp 1 (2017).

[12] O. Bou Matar, N. Gasmi, H. Zhou, M. Goueygou, and A. Talbi, J. Acoust. Soc. Am. 133, 1415 (2013)]. 